\title{
Method of Evaluating and Predicting Traffic State of Highway Network Based on Deep Learning
}

\author{
Jiayu Liu $\left(\mathbb{D},{ }^{1}\right.$ Xingju Wang $\left(\mathbb{D},{ }^{1,2}\right.$ Yanting Li $\left(\mathbb{D},{ }^{1}\right.$ Xuejian Kang $\left(\mathbb{D},{ }^{1,2}\right.$ and Lu Gao $\mathbb{D}^{3}$ \\ ${ }^{1}$ School of Traffic and Transportation, Shijiazhuang Tiedao University, Shijiazhuang, China \\ ${ }^{2}$ State Key Laboratory of Mechanical Behavior and System Safety of Traffic Engineering Structures, \\ Shijiazhuang Tiedao University, Shijiazhuang, China \\ ${ }^{3}$ Department of Construction Management, University of Houston, 4730 Calhoun Road No. 300 Houston, Houston, \\ TX 77204-4021, USA
}

Correspondence should be addressed to Xingju Wang; wangxingju@stdu.edu.cn

Received 16 July 2020; Revised 24 November 2020; Accepted 17 March 2021; Published 24 March 2021

Academic Editor: Haneen Farah

Copyright $(2021$ Jiayu Liu et al. This is an open access article distributed under the Creative Commons Attribution License, which permits unrestricted use, distribution, and reproduction in any medium, provided the original work is properly cited.

\begin{abstract}
The accurate evaluation and prediction of highway network traffic state can provide effective information for travelers and traffic managers. Based on the deep learning theory, this paper proposes an evaluation and prediction model of highway network traffic state, which consists of a Fuzzy C-means (FCM) algorithm-based traffic state partition model, a Long Short-Term Memory (LSTM) algorithm-based traffic state prediction model, and a K-Means algorithm-based traffic state discriminant model. The highway network in Hebei Province is employed as a case study to validate the model, where the traffic state of highway network is analyzed using both predicted data and real data. The dataset contains 536,823 pieces of data collected by 233 continuous observation stations in Hebei Province from September 5, 2016, to September 12, 2016. The analysis results show that the model proposed in this paper has a good performance on the evaluation and prediction of the traffic state of the highway network, which is consistent with the discriminant result using the real data.
\end{abstract}

\section{Introduction}

With the increasing of vehicle ownership, the demand for transportation has also expanded rapidly, which not only brings great pressure to the highway traffic system, but also causes a series of congestion problems and safety issues. The 2019 Hebei Statistical bulletin on national economic and social development indicates that the mileage open to traffic increased by $1.9 \%$ in the province, but the vehicle ownership increased by $7.4 \%$ year-over-year [1]. Therefore, it is particularly essential to be able to evaluate and predict the traffic state of the highway network accurately in real time. Traffic managers can develop prevention strategies in advance to effectively avoid the occurrence of traffic congestion and prevent the further expansion of the impact of congestion. How to realize the accurate evaluation and prediction of traffic state is the most important task faced by traffic managers and traffic problem researchers.
Appropriate indicators need to be selected for the evaluation of traffic state, mainly including service level [2], traffic flow parameters [3-5], and travel time [6]. In addition, a large number of scholars have studied the evaluation method of traffic state. The existing researches on the traffic state evaluation of road primarily focus on the intersection [7], road [8], and road network [9, 10]. For transportation node, most studies focus on urban roads and use major intersections as the research object. Xu et al. have conducted in-depth research on the evaluation of road traffic state. They used various theories and methods to estimate and predict the road traffic state [11-14]. In addition, D.W. Xu et al. proposed a novel deep learning framework for estimating road traffic state [15]. As the development of big data [16] and machine learning [17] advances, more methodologies will be applied to the evaluation of highway network traffic state. B. Hillel used cellular phone service provider information to measure traffic speed and travel time [18]. Based 
on a large amount of floating car data, G. Fusco et al. made short-term forecasts of traffic speed and carried out comparative analysis using a variety of methods [19]. A. Wu et al. adopted floating car data to identify the traffic state of signalized intersections [20]. M. Wang et al. used a large cell phone network data to estimate traffic flow [21]. A large number of loop detectors are installed in the highway network to collect traffic information. These data can also be used to analyze traffic state $[22,23]$. In addition, based on machine learning theory, neural networks have been widely applied to traffic flow prediction [24, 25]. J. Yu et al. presented a graph convolutional generative autoencoder model to estimate traffic speed in real time [26]. I. Laña et al. presented a method with an adaptation mechanism for longterm urban traffic volume forecasting [27].

The previously related research is mainly divided into two aspects including predicting traffic flow parameters, such as traffic volume, density, speed, and saturation, and evaluating the road traffic state. Little attention is paid to the evaluation and prediction of road network traffic state. Taking highway network as the research object, this study aims to conduct the evaluation and prediction model of highway network traffic state based on deep learning. The evaluating and predicting traffic state of highway network can comprehensively reflect the traffic condition of the entire highway network. This study could be useful for future policy making.

\section{Methods}

The traffic state of the highway network is divided into four categories, namely, severe congestion state, congestion state, general state, and smooth state. The speed and density of traffic flow are selected as evaluation indicators to develop the proposed evaluation and prediction model of highway network traffic state based on deep learning. The model mainly includes the partition model of traffic state based on the Fuzzy C-means (FCM) algorithm, the prediction model of traffic state based on the Long Short-Term Memory (LSTM) algorithm, and the discriminant model of traffic state based on K-Means algorithm.

\subsection{The Partition Model of Highway Network Traffic State} Based on FCM Algorithm. The traffic state of the highway network has certain fuzziness. The speed and density of traffic flow are used as evaluation indicators, and the FCM algorithm is used to evaluate the traffic state of the highway network. The FCM algorithm can well analyze the attribute characteristics of big data in practical problems, and it uses the membership function to cluster [28].

The FCM algorithm divides the $n$ vectors $\mathbf{x}_{i}(i=1,2, \ldots, n)$ into $c$ classes and calculates the cluster centers for each class. For $\forall \mathbf{x}_{i}=\left(v_{i}, k_{i}\right), v_{i}, k_{i}$ represent the speed and density of the ith sample, respectively, and $1<c<n$. The objective function defined by the membership function is as follows:

$$
\begin{aligned}
& \min \{J(\mathbf{U}, \mathbf{A})\}=\min \left\{\sum_{j=1}^{c} \sum_{i=1}^{n}\left(u_{i j}\right)^{m}\left(d_{i j}\right)^{2}\right\}, \\
& \text { s.t. }\left\{\begin{array}{l}
0 \leq u_{i j} \leq 1, \\
\sum_{j=1}^{c} u_{i j}=1,
\end{array}\right.
\end{aligned}
$$

where $J(\mathbf{U}, \mathbf{A})$ is the weighted value of the sample to each cluster center of the highway network traffic state; $u_{i j}$ is the membership function of the ith sample for the $j$ class, and $1 \leq j \leq c, 1 \leq i \leq n ; \mathbf{A}=\left[\mathbf{a}_{1}, \ldots, \mathbf{a}_{j}, \ldots, \mathbf{a}_{c}\right]$ is the $2 \times c$ order cluster center matrix; $\mathbf{a}_{j}$ is the eigenvector of the $j t h$ cluster center of the highway network traffic state and $\mathbf{a}_{j}=\left(v_{j}, k_{j}\right)$; $m$ is the fuzzy weighted index; $\left(d_{i j}\right)^{2}=\left\|\mathbf{x}_{i}-\mathbf{a}_{j}\right\|$ is the Euclidean distance from the $i$ th sample to $\mathbf{a}_{j}$; and $\mathbf{U}=\left\{u_{i j}\right\}$ is the $c \times n$ order fuzzy classification matrix.

Construct the following formula by using Lagrange multiplication:

$$
F=\sum_{j=1}^{c}\left(u_{i j}\right)^{m}\left(d_{i j}\right)^{2}+\lambda\left(\sum_{i=1}^{c} u_{i j}-1\right) .
$$

Taking the derivatives for all input variables, we can get the following formula:

$$
\begin{aligned}
& u_{i j}=\frac{1}{\sum_{r=1}^{c}\left(d_{i j} / d_{k j}\right)^{2 / m-1}}, \\
& \mathbf{a}_{j}=\frac{\sum_{k=1}^{n}\left(u_{j k}\right)^{m} x_{k}}{\sum_{k=1}^{n}\left(u_{j k}\right)^{m}} .
\end{aligned}
$$

\subsection{Algorithm Implementation}

(1) Initialization. Give the iterative standards $\varepsilon>0$, set $b$ as the number of iterations, and let $b=0$. The membership matrix is calculated as

$$
u_{i j}^{(b)}=\frac{1}{\sum_{r=1}^{c}\left(d_{i j}^{(b)} / d_{r j}^{(b)}\right)^{2}} .
$$

If $i, r$ exist, so that $d_{i r}^{(b)}=0$, then there is $u_{i r}^{(b)}=1$, and $j \neq r, u_{i r}^{(b)}=0$.

(2) The iterative operation of the cluster center is

$$
\mathbf{a}_{j}^{(b+1)}=\frac{\sum_{i=1}^{n}\left(u_{i j}^{(b)}\right)^{2} \mathbf{x}_{i}}{\sum_{i=1}^{n}\left(u_{i j}^{(b)}\right)^{2}}, \quad j=1,2, \ldots, c .
$$

(3) Use a matrix norm $\|\cdot\|$ to compare $\mathbf{a}^{(b+1)}$ and $\mathbf{a}^{(b)}$; if $\left\|\mathbf{a}^{(b+1)}-\mathbf{a}^{(b)}\right\| \leq \varepsilon$, stop iteration; otherwise, let $b=b+1$, and then turn to the second step.

(4) The cluster center matrix of the highway network traffic state is finally obtained as $\mathbf{A}=\left[\mathbf{a}_{1}, \ldots, \mathbf{a}_{j}, \ldots, \mathbf{a}_{c}\right]$. 
The driving speed and density of the vehicle on the highway network can well reflect the traffic state of the highway network. The faster the driving speed of the vehicle, the smaller the density, the better the highway condition, and the smoother the traffic state of the highway network. According to the above algorithm, the cluster center matrix and the classification result of the traffic state of the highway network can be obtained.

\subsection{The Prediction Model of Highway Network Traffic State} Based on LSTM Algorithm. LSTM is a long-term and shortterm memory neural network. The model can be trusted for a long time, which is suitable for processing and predicting time series data and important events with relatively long delays. It has been used in many fields of science and technology. The model training methods proposed in this paper include forward calculation using activation function and backward estimation using the backpropagation through time (BPTT) according to the loss function [29].

The data are preprocessed by Z-score normalization method before training. The processed function has a mean value of 0 and a standard deviation of 1 as shown in (6). After outputting the predicted result, the data need to be antinormalized to obtain the true predicted values of the traffic state evaluation indicators. The antinormalization formula is shown in (7):

$$
\begin{aligned}
x^{*} & =\frac{x-\mu}{\sigma}, \\
x & =x^{*} * \sigma+\mu,
\end{aligned}
$$

where $\mu$ is the mean of all sample data, and $\sigma$ is the standard deviation of all sample data.

In order to verify the accuracy of the prediction result and the feasibility of the prediction model, it is necessary to perform error analysis on this result. The relative error formula of each predicted point is shown in (8). The Mean Absolute Percentage Error (MAPE) and Root Mean Square Error (RMSE) of the highway or the highway network are calculated using (9) and (10), respectively.

$$
\begin{aligned}
\varepsilon_{i} & =\frac{y_{i}^{*}-y_{i}}{y_{i}}, \\
\text { MAPE } & =\frac{1}{N} \sum_{i=1}^{N} \frac{\left|y_{i}^{*}-y_{i}\right|}{y_{i}} \times 100 \%, \\
\text { RMSE } & =\sqrt{\frac{1}{N} \sum_{i=1}^{N}\left(y_{i}^{*}-y_{i}\right)^{2},}
\end{aligned}
$$

where $\varepsilon_{i}$ is the relative error between the predicted value and the real value of the $i$ th sample data, $y_{i}$ is the real value of the $i$ th sample data, $y_{i}^{*}$ is the predicted value of the $i$ th sample data, and $N$ is the number of sample data of the traffic state evaluation indicators.
2.4. The Discriminant Model of Highway Network Traffic State Based on K-Means Algorithm. The K-Means algorithm is an unsupervised clustering algorithm, and the basic idea of the algorithm is that, for a given sample set, it is divided into multiple clusters according to the distance between the samples. Using the Euclidean distance as the measure of similarity, the shortest distance between each sample and each cluster center can be found. Then, we can know the traffic state of each sample. The specific formula is as follows:

$$
G\left(x_{p}\right)=\min _{k=1}^{c}\left\{D\left(\left(v_{p}, k_{p}\right),\left(v_{a_{j}}, k_{a_{j}}\right)\right)\right\},
$$

where

$$
D\left(\left(v_{p}, k_{p}\right),\left(v_{a_{j}}, k_{a_{j}}\right)\right)=\sqrt{\beta_{1}\left(v_{p}-v_{a_{j}}\right)^{2}+\beta_{2}\left(k_{p}-k_{a_{j}}\right)^{2}},
$$

where $x_{p}$ is the $p$ th highway traffic state prediction sample; $v_{p}$ and $k_{p}$ are, respectively, the speed and density parameters of the pth highway traffic state prediction sample; $a_{j}$ is the cluster center of the $j$ th class of the highway traffic state; $v_{a_{j}}$ and $k_{a_{j}}$ are the speed and density of $a_{j}$, respectively; $\beta_{1}$ and $\beta_{2}$ are the weight of the influence of speed and density, respectively, on the classification of the traffic state, and this paper considers the influence as the same, namely, $0 \leq \beta_{1} \leq 1,0 \leq \beta_{2} \leq 1, \beta_{1}+\beta_{2}=1$.

In order to evaluate the traffic state of the highway network, the average speed and density of all highways need to be calculated. Based on the K-means algorithm, the average traffic state of the highway network can be obtained.

\section{Case Study}

3.1. Dataset. The dataset is collected by 233 continuous observation stations from September 5, 2016, to September 12,2016 . The original dataset has certain quality problems, so the dataset is cleaned and processed. The dataset is the comprehensive traffic flow information by direction, lane, and vehicle mode. The observation interval is set to be five minutes, and each observation station collects 288 pieces of data per day. Thus, 536,832 pieces of data are collected from 233 observation stations. The layout of the observation stations in the highway network is shown in Figure 1. The main attribute information of the observation station is shown in Table 1. The data for one day the at Babaihu observation station are listed in Table 2. There is an observation station on each highway, and the observation data of the observation station represent the traffic information of the highway on which it is located.

3.2. Partition of Highway Network Traffic State. The highway network is composed of different highways, and each highway has its specific design speed. Therefore, before using the FCM algorithm for clustering, the traffic flow speed and density of the highway network should be standardized. The standardized formula is as follows: 


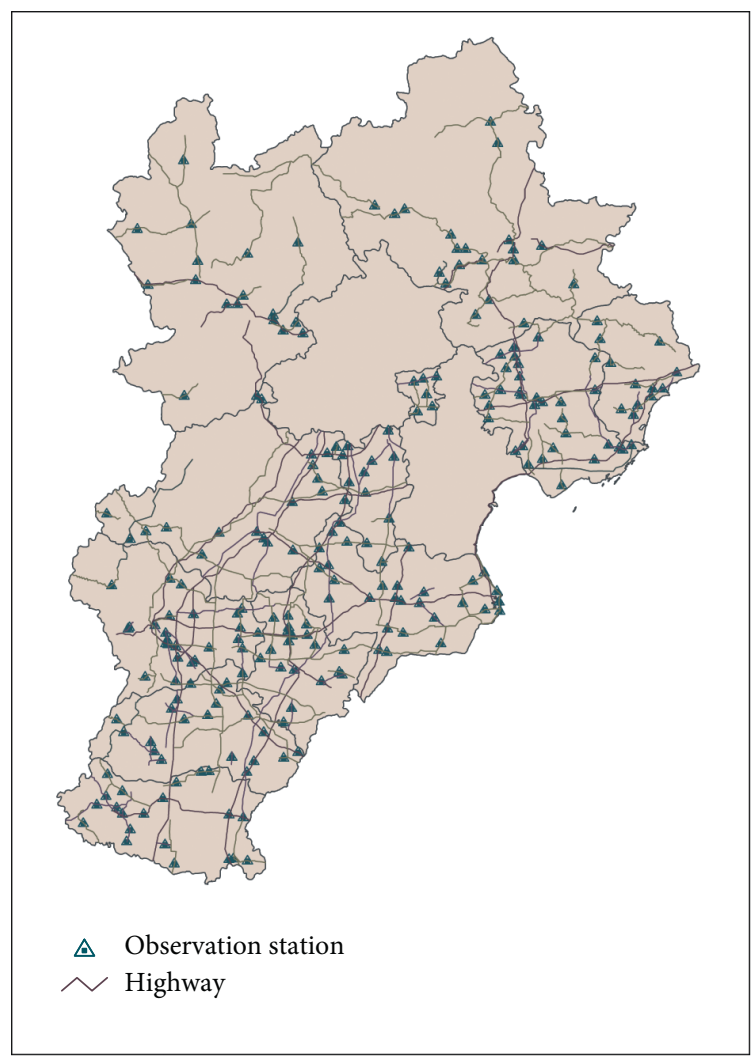

Figure 1: The layout of the observation stations in the highway network.

TABLE 1: The main attribute information of the observation station.

\begin{tabular}{lccccc}
\hline Index & Year & Observation station name & Observation station type & Investigation method & Design speed $(\mathrm{km} / \mathrm{h})$ \\
\hline 1 & 2016 & Qingxianzhuxianzhan & Continuous & Equipment observation & 120 \\
2 & 2016 & Cangzhoubei & Continuous & Equipment observation & 120 \\
3 & 2016 & Cangzhouxi & Continuous & Equipment observation & 120 \\
4 & 2016 & Xiadian & Continuous & Equipment observation & 80 \\
5 & 2016 & Dadingfu & Continuous & Equipment observation & 80 \\
6 & 2016 & Liangjiadian & Continuous & Equipment observation & $\ldots$ \\
$\ldots$ & $\ldots$ & $\ldots$ & $\ldots$ & $\ldots$ & 80 \\
228 & 2016 & Pianqiaozi & Continuous & Equipment observation & 80 \\
229 & 2016 & Dongying & Continuous & Equipment observation & 60 \\
230 & 2016 & Taipingzhuang & Continuous & Equipment observation & 40 \\
231 & 2016 & Mujiangkou & Continuous & Equipment observation & \\
232 & 2016 & Yeheqiaoyanghuzhongxin & Continuous & Equipment observation & 40 \\
233 & 2016 & Erbaozi & Continuous & Equipment observation & 30 \\
\hline
\end{tabular}

TABLE 2: Babaihu observation station data.

\begin{tabular}{|c|c|c|c|c|c|c|}
\hline Index & Observation station & Hour & Minute & Flow volume $[\mathrm{pcu}]$ & Speed $(\mathrm{km} / \mathrm{h})$ & Density[pcu $/ \mathrm{km}]$ \\
\hline 1 & Babaihu & 1 & 0 & 8 & 25.0000 & 10.4800 \\
\hline 2 & Babaihu & 1 & 5 & 41 & 24.8750 & 10.5327 \\
\hline 3 & Babaihu & 1 & 10 & 21 & 24.8333 & 10.5503 \\
\hline 4 & Babaihu & 1 & 15 & 25 & 22.3333 & 11.7313 \\
\hline 5 & Babaihu & 1 & 20 & 22 & 22.5000 & 11.6444 \\
\hline 6 & Babaihu & 1 & 25 & 16 & 22.3750 & 11.7095 \\
\hline$\ldots$ & $\ldots$ & $\ldots$ & $\ldots$ & $\ldots$ & $\ldots$ & $\ldots$ \\
\hline 283 & Babaihu & 24 & 30 & 17 & 24.4000 & 11.5984 \\
\hline 284 & Babaihu & 24 & 35 & 7 & 22.7500 & 12.4396 \\
\hline 285 & Babaihu & 24 & 40 & 14 & 22.0000 & 12.8636 \\
\hline 286 & Babaihu & 24 & 45 & 38 & 23.5000 & 12.0426 \\
\hline 287 & Babaihu & 24 & 50 & 12 & 26.0000 & 10.8846 \\
\hline 288 & Babaihu & 24 & 55 & 39 & 27.2500 & 10.3853 \\
\hline
\end{tabular}




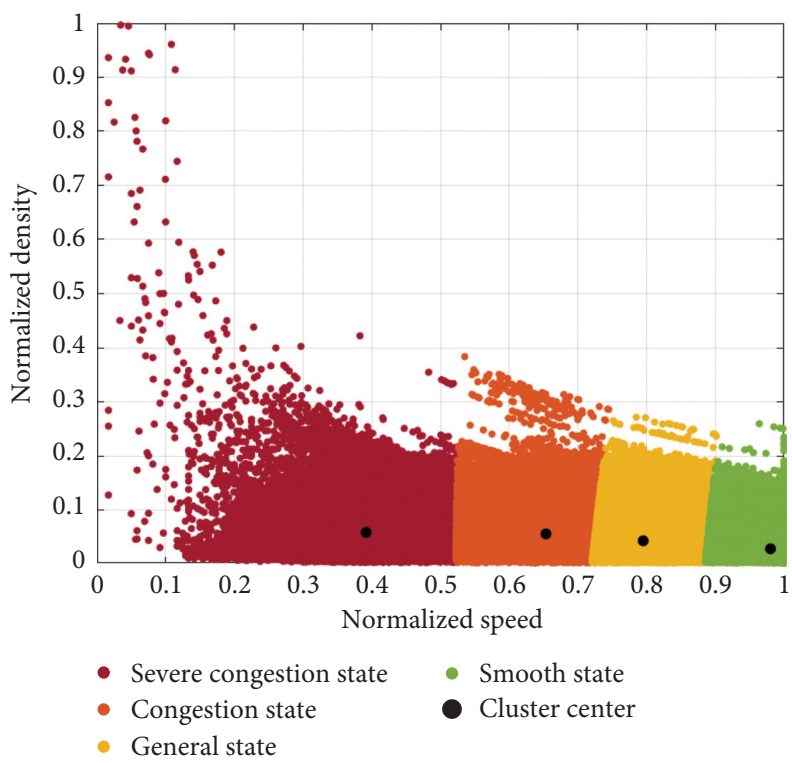

Figure 2: Highway network classification result.
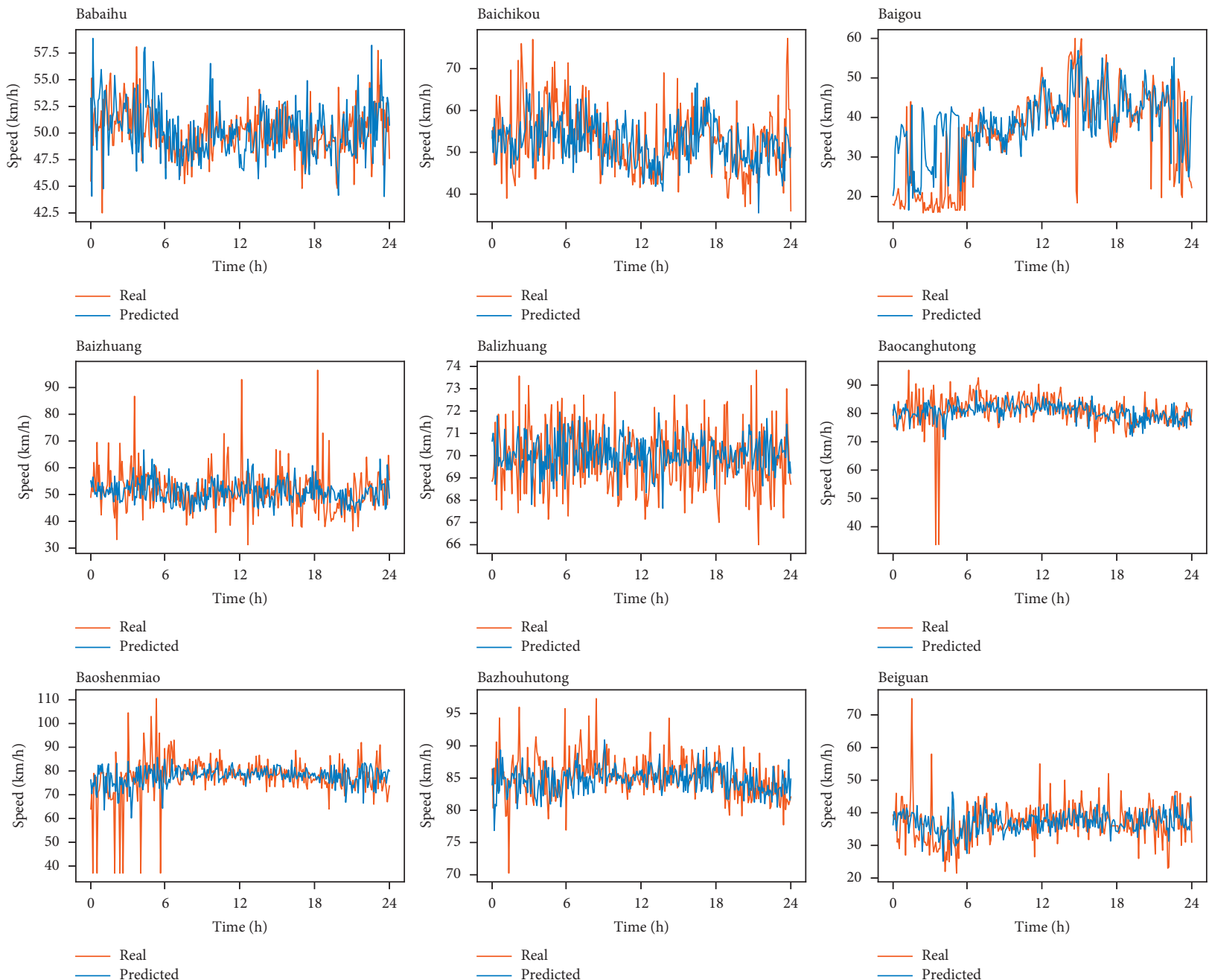

Figure 3: Comparison of predicted speed and true speed in 9 observation stations. 

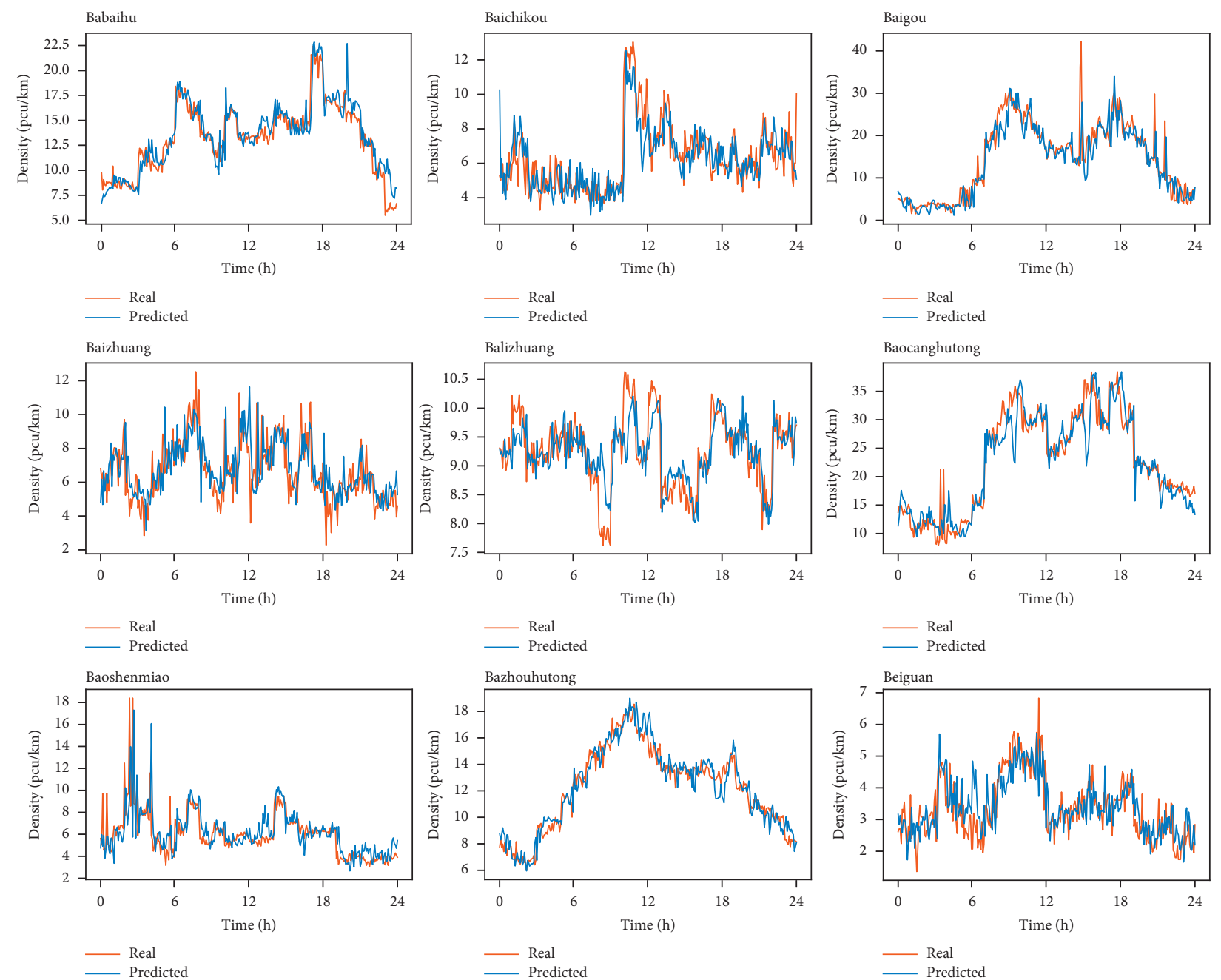

FIgURE 4: Comparison of predicted density and true density in 9 observation stations.

$$
\begin{aligned}
& v_{i}^{\prime}=\frac{v_{i}-v_{\min }}{v_{\max }-v_{\min }}, \\
& k_{i}^{\prime}=\frac{k_{i}-k_{\min }}{k_{\max }-k_{\min }} .
\end{aligned}
$$

When using FCM algorithm to classify traffic state of the highway network, the model parameters need to be selected first. As discussed previously, the number of clusters $c=4$. For fuzzy weighted index $m$, numerous studies have been conducted, and it is widely accepted that, for general classification, $m=2$ is rational [30]. Speed and density data collected by 233 observation stations from September 5, 2016, to September 11, 2016, are studied. The collected dataset contains 469,728 pieces of data. The calculated cluster center is shown below.

$$
A=\left[\begin{array}{ll}
0.3919 & 0.0584 \\
0.6535 & 0.0558 \\
0.7951 & 0.0430 \\
0.9804 & 0.0282
\end{array}\right] .
$$

The first column of matrix $A$ shows the normalized speed value of the cluster centers. The second column shows the normalized density value of the cluster centers. Since the faster the speed, the smaller the density, and the better the traffic, the four rows in matrix $A$ show cluster centers with severe congestion state, congestion state, general state, and smooth state from top to bottom, respectively. The clusters found by FCM are shown in Figure 2.

3.3. Prediction of Highway Network Traffic State. The LSTM algorithm is utilized to develop the short-term prediction. The data from September 5, 2016, to September 11, 2016, are the training set and the data from September 12, 2016, are the testing set. The speed and density of each observation station are predicted separately, and the prediction errors are calculated. The comparison of predicted speed and true speed in some observation stations is shown in Figure 3. And the comparison of predicted density and true density in some observation stations is shown in Figure 4. According to (9) and (10), the MAPE and RMSE of the speed and density of 233 observation stations are obtained. The resulting errors 


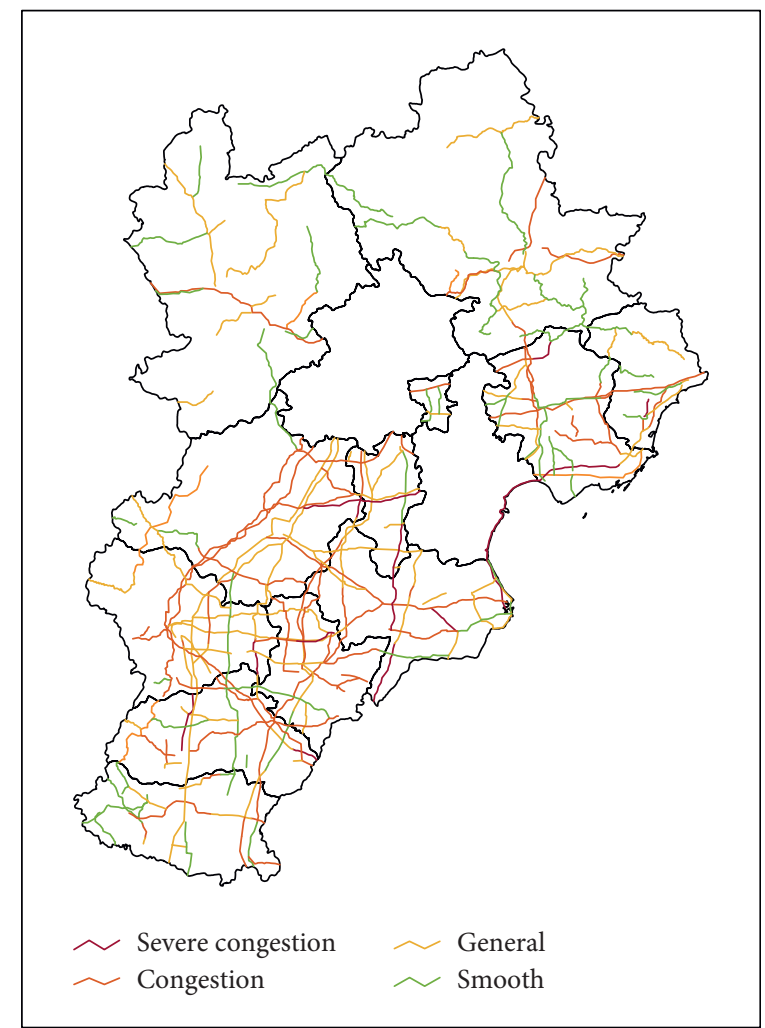

Figure 5: The predicted average traffic state of each highway.

are extremely small, indicating that the predicted value is relatively accurate.

$$
\begin{aligned}
\operatorname{MAPE}(\text { speed }) & =8.7892, \\
\operatorname{RMSE}(\text { speed }) & =0.1264, \\
\operatorname{MAPE}(\text { density }) & =2.0948, \\
\operatorname{RMSE}(\text { density }) & =0.1824 .
\end{aligned}
$$

3.4. Discriminant of Highway Network Traffic State. The speed and density of each highway are determined by the observations of the observation station where it is located. Before judging the traffic state of each highway, the predicted speed and density values should be normalized according to (13) and (14). The traffic state of each highway is discriminated based on the K-means algorithm. Then, calculating the average speed and density of all highways can obtain the average traffic state of the highway network. The discriminant result of the average traffic state of each highway is shown in Figure 5. And the normalized average predicted speed and density of the highway network are 0.7719 and 0.0467 , respectively. Therefore, according to the proposed evaluation and prediction model of highway network traffic state based on deep learning, on September 12, 2016, the average traffic state of the highway network constituted by 233 highways is in general state.

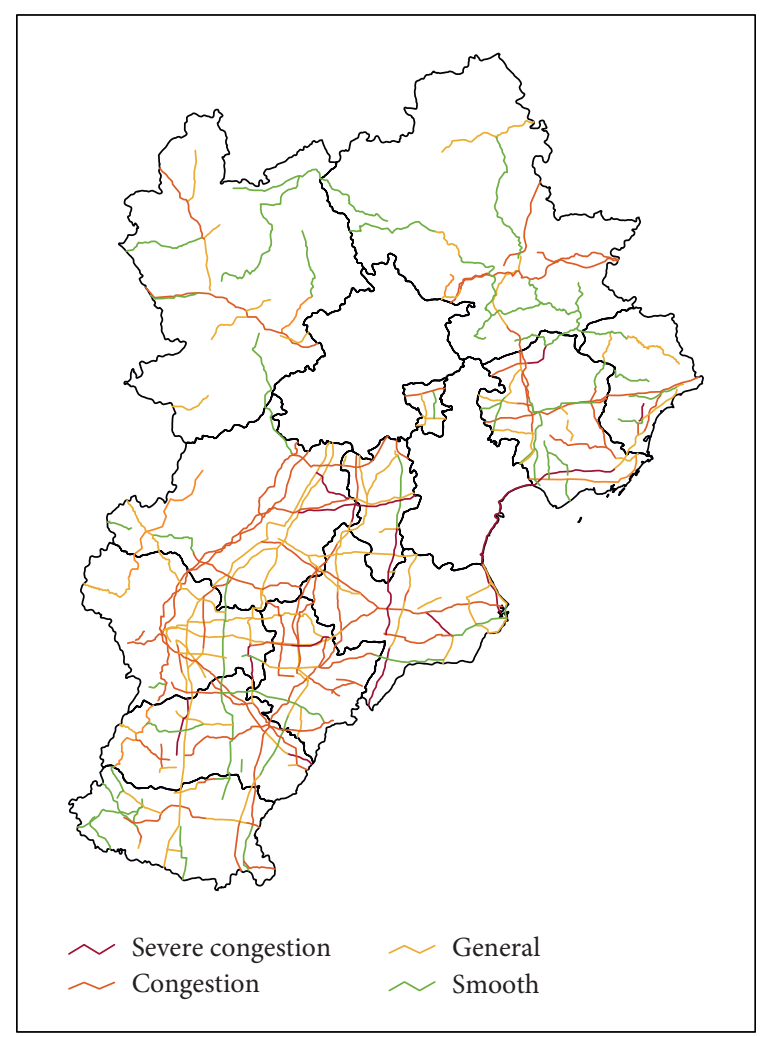

FIgURE 6: The real average traffic state of each highway. 
TABLE 3: The proportion of each traffic state category.

\begin{tabular}{|c|c|c|c|c|}
\hline $\begin{array}{l}\text { Traffic state } \\
\text { percentage }\end{array}$ & Severe congestion state (\%) & Congestion state $(\%)$ & General state $(\%)$ & Smooth state $(\%)$ \\
\hline The proportion of each predicted traffic sta & 5.58 & 30.47 & 38.32 & 25.75 \\
\hline The proportion of each real traffic state & 6.01 & 30.90 & 37.77 & 25.32 \\
\hline
\end{tabular}

3.5. Analysis of Evaluation Results. To prove the accuracy of the proposed model, the true average traffic state of the highway network on September 12, 2016, needs to be analyzed. First, the true average speed and density of each highway on September 12, 2016, are calculated and normalized, and then the average traffic state of each highway is discriminated, which is shown in Figure 6. The true normalized average speed and density of the highway network are 0.7710 and 0.0471 , respectively. And the predicted and true traffic state are consistent, and both are in general state. The predicted and real average traffic states of each highway are compared, and the proportion of each traffic state in the highway network is shown in Table 3 . The results show that congestion state and general state account for the largest proportion. About $6 \%$ of highways are in severe congestion state, indicating that the speed of vehicles is restricted to a certain extent. The operating conditions are not good, and the highway is in high pressure. Such problem could be caused by the imbalance between supply and demand of existing highway infrastructure and traffic demand caused. The traffic management departments should pay attention to the reconstruction and development of these highways. Approximately $30 \%$ of the highways are in congestion state, and these highways should also be focused on. Reasonable traffic organization plans and control measures should be formulated in advance to alleviate traffic congestion. When the highways are in general state and smooth state, it means that the vehicles can travel at a faster speed and are in good operating condition. And in the highway network, more than $50 \%$ of the highways are not congested. It shows that the overall layout of the highway network is relatively reasonable, and the traffic condition is good. The evaluation and prediction of the traffic state of the highway network can play a role in prevention and planning in advance.

\section{Conclusions}

This paper proposes an evaluation and prediction model of highway network traffic state based on deep learning. The model consists of a FCM algorithm-based traffic state partition model, a LSTM algorithm-based traffic state prediction model, and a K-Means algorithm-based traffic state discriminant model. In this study, the traffic state of the highway network is divided into four categories: severe congestion state, congestion state, general state, and smooth state. The speed and density of traffic flow are selected as evaluation indicators. To validate the effectiveness of the proposed model, the traffic flow data of 233 observation stations in Hebei Province from September 5, 2016, to September 12, 2016, are collected. The observation interval is
5 minutes, and the total of data is 536,823 pieces. The main findings are concluded as follows:

On September 12, 2016, the predicted average traffic state of the highway network constituted by 233 highways is in general state, which is consistent with the real traffic state. The overall layout of the highway network is relatively reasonable, and the supply and demand of transportation are basically balanced.

The highway network traffic state is an objective reflection of the traffic condition. The evaluation and prediction of highway network traffic state provide the dynamic characteristic of traffic change. Based on this information, travelers can make their travel plans better and improve their travel efficiency. Traffic managers can also work out better traffic organization plans in advance to reduce traffic congestion.

\section{Data Availability}

The data used to support the findings of this study are available from the corresponding author upon request.

\section{Conflicts of Interest}

The authors declare that there are no conflicts of interest regarding the publication of this paper.

\section{Acknowledgments}

This work was supported by the Scientific Research Foundation, Education Department of Hebei Province of China (ZD2021336). Moreover, this work was further sponsored by the Natural Science Foundation of Hebei Province in China (E2019210305) and Soft Science Research Project of Hebei Province (20557673D) for funding.

\section{References}

[1] Hebei Bureau of Statistics, http://www.hetj.gov.cn/hetj/app/ tjgb/101591084423716.html Hebei 2019 Provincial Economy and Social Development Statistical Report, 2019.

[2] A. C. Tay and H. H. Lee, "Traffic condition with road upgrading during construction and operation stages based on level-of-service (LOS)," IOP Conference Series Materials Science and Engineering, vol. 344, no. 1, Article ID 012018, 2018.

[3] S. Toru, M. B. Alexandre, K. Takahio et al., "Traffic state estimation on highway: a comprehensive survey," Annual Reviews in Control, vol. 43, pp. 128-151, 2017.

[4] H.-Y. Yao, J. Wang, Y. Chen, Q. Tu, and B. Cheng, "Evaluation method for traffic operation status on ordinary highway roads," Journal of Highway and Transportation Research and Development (English Edition), vol. 10, no. 2, pp. 73-78, 2016. 
[5] A. Zeroual, F. Harrou, Y. Sun et al., "Road traffic density estimation and congestion detection with a hybrid observerbased strategy," Sustainable Cities and Society, vol. 46, 2019.

[6] E. Ugnenko, E. Uzhvieva, Y. Voronova et al., "Simulation of traffic flows on the road network of urban area," Procedia Engineering, vol. 134, pp. 153-156, 2016.

[7] H.-T. Zhao, X.-R. Liu, X.-X. Chen, and J.-C. Lu, "Cellular automata model for traffic flow at intersections in internet of vehicles," Physica A: Statistical Mechanics and Its Applications, vol. 494, pp. 40-51, 2018.

[8] Y. Kawasaki, Y. Hara, M. Kuwahara et al., "Traffic state estimation on a two-dimensional network by a state-space model," Transportation Research Part C: Emerging Technologies, vol. 113, pp. 176-192, 2020.

[9] A. Jayasinghe, K. Sano, C. C. Abenayake, and P. K. S. Mahanama, "A novel approach to model traffic on road segments of large-scale urban road networks," MethodsX, vol. 6, pp. 1147-1163, 2019.

[10] G. Zhu, K. Song, P. Zhang, and L. Wang, "A traffic flow state transition model for urban road network based on Hidden Markov Model," Neurocomputing, vol. 214, no. 19, pp. 567-574, 2016.

[11] D.-W. Xu, H.-H. Dong, L.-M. Jia, and Y. Tian, "Road traffic states estimation algorithm based on matching of regional traffic attracters," Journal of Central South University, vol. 21, no. 5, pp. 2100-2107, 2014.

[12] D. W. Xu, H. H. Dong, H. J. Li, L. M. Jia, and Y. J. Feng, "The estimation of road traffic states based on compressive sensing," Transportmetrica B: Transport Dynamics, vol. 3, no. 2, pp. 131-152, 2015.

[13] D.-W. Xu, Y.-D. Wang, L.-M. Jia, Y. Qin, and H.-H. Dong, "Real-time road traffic state prediction based on ARIMA and Kalman filter," Frontiers of Information Technology \& Electronic Engineering, vol. 18, no. 2, pp. 287-302, 2017.

[14] D. Xu, Y. Wang, P. Peng, S. Beilun, Z. Deng, and H. Guo, "Real-time road traffic state prediction based on kernelKNN," Transportmetrica A: Transport Science, vol. 16, no. 1, pp. 104-118, 2018.

[15] D. Xu and P. Peng, "A novel deep learning framework for road traffic state estimation," Transportation Research Part C: Emerging Technologies, vol. 117, 2020.

[16] S. Amini, I. Gerostathopoulos, C. Prehofer et al., "Big data analytics architecture for real-time traffic control," in Proceedings of the IEEE International Conference on Models and Technologies for Intelligent Transportation Systems (MT-ITS), pp. 710-715, Naples, Italy, June 2017.

[17] J. Wang, R. Chen, and Z. He, "Traffic speed prediction for urban transportation network: a path based deep learning approach," Transportation Research Part C: Emerging Technologies, vol. 100, pp. 372-385, 2019.

[18] H. Bar-Gera, "Evaluation of a cellular phone-based system for measurements of traffic speeds and travel times: a case study from Israel," Transportation Research Part C: Emerging Technologies, vol. 15, no. 6, pp. 380-391, 2007.

[19] G. Fusco, C. Colombaroni, N. Isaenko et al., "Short-term speed predictions exploiting big data on large urban road networks," Transportation Research Part C: Emerging Technologies, vol. 73, pp. 183-201, 2016.

[20] A. Wu, W. Yin, X. Yang et al., "Research on the real-time traffic status identification of signalized intersections based on floating car data," Procedia - Social and Behavioral Sciences, vol. 96, pp. 1578-1584, 2013.

[21] M.-H. Wang, S. D. Schrock, and T. Mulinazzi, "Estimating dynamic origin-destination data and travel demand using cell phone network data," International Journal of Intelligent Transportation Systems Research, vol. 11, no. 2, pp. 76-86, 2013.

[22] B. Li, "Recursive estimation of average vehicle time headway using single inductive loop detector data," Transportation Research Part B: Methodological, vol. 46, no. 1, pp. 85-99, 2012.

[23] J. Li, H. J. Van Zuylen, G. Wei et al., "Diagnosing and interpolating loop detector data errors with probe vehicle data," Transportation Research Record, vol. 2423, pp. 61-67, 2014.

[24] X. Ma, Z. Tao, Y. Wang, H. Yu, and Y. Wang, "Long shortterm memory neural network for traffic speed prediction using remote microwave sensor data," Transportation Research Part C: Emerging Technologies, vol. 54, pp. 187-197, 2015.

[25] Z. Zhao, W. Chen, X. Wu, P. C. Y. Chen, and J. Liu, "LSTM network: a deep learning approach for short-term traffic forecast," Iet Intelligent Transport Systems, vol. 11, no. 2, pp. 68-75, 2017.

[26] J. J. Q. Yu and J. Gu, "Real-time traffic speed estimation with graph convolutional generative autoencoder," IEEE Transactions on Intelligent Transportation Systems, vol. 20, no. 10, pp. 3940-3951, 2019.

[27] I. Laña, J. L. Lobo, E. Capecci, J. Del Ser, and N. Kasabov, "Adaptive long-term traffic state estimation with evolving spiking neural networks," Transportation Research Part C: Emerging Technologies, vol. 101, pp. 126-144, 2019.

[28] N. R. Pal and J. C. Bezdek, "On cluster validity for the fuzzy c-means model," IEEE Transactions on Fuzzy Systems, vol. 3, no. 3, pp. 370-379, 1995.

[29] F. A. Gers, J. Schmidhuber, and F. Cummins, "Learning to forget: continual prediction with LSTM," Neural Computation, vol. 12, no. 10, pp. 2451-2471, 2000.

[30] Z. Cheng, W. Wang, J. Lu, and X. Xing, "Classifying the traffic state of urban expressways: a machine-learning approach," Transportation Research Part A: Policy and Practice, vol. 137, pp. 411-428, 2020. 